\title{
Die Ästhetik des Bösen als „postmodernes“ Stilprinzip? Theologisch-ethische Randbemerkungen zur TV-Serie Twin Peaks ${ }^{1}$
}

\author{
von Walter Lesch
}

„Seit die Menschen nicht mehr an Gott glauben, glauben sie nicht etwa an nichts mehr, sondern an alles." Gilbert Keith Chesterton ${ }^{2}$

\section{Vorbemerkungen zum theologischen Interesse am Film}

Die Begegnungen zwischen Film und Theologie haben trotz vielfacher Bemühungen um größere Selbstverständlichkeit immer noch den Reiz des Exotischen. Warum um alles in der Welt sollten FilmemacherInnen daran interessiert sein, mit TheologInnen über Produkte und Projekte der Filmkunst ins Gespräch zu kommen? Ausnahmen bestätigen wie immer die Regel. Doch insgesamt ist zu vermuten, daß ein großer Teil der kommerziellen und künstlerischen Filmbranche mit der Theologie nicht viel im Sinn hat. Andererseits soll nicht geleugnet werden, daß das Thema "Religion im Film“ nicht nur für einige Spezialisten relevant ist und vor allem nicht auf den Spezialfall des explizit „religiösen“ Films reduziert werden kann. Sinnfragen sind unvermeidbar im internationalen Filmgeschäft wie in jedem Segment der Kultur präsent; und ethische Fragen können ohnehin kaum umgangen werden, insofern Filme im Normalfall etwas mit Handlungskonstruktionen zu tun haben, über deren Logik und Bewertung ethisch nachgedacht werden kann, wenn auch nicht muß.

Ich kann verstehen, wenn schon an dieser Stelle Unmut aufkommt, weil der Eindruck entstehen könnte, daß eine sich modern gebende Theologie wieder einmal verständnisvoll deklamiert, ihr sei nichts Menschliches fremd; sie sei prinzipiell an jedem Kulturprodukt interessiert und werde folglich gewissenhaft und unbefangen prüfen, wie sich eine Brücke zur christlichen Tradition bauen lasse. Ich muß gestehen, daß mich das Dialoggefasel einiger theologischer Dünnbrettbohrer nicht sehr beeindruckt. Wenn das sogenannte Gespräch mit dem Film nur gesucht wird, um die eigene Modernität und Liberalität unter Beweis zu stellen oder um dem persönlichen Freizeitinteresse einen professionellen Anstrich zu geben, dann ist es vielleicht besser, wenn entsprechende „Dialogprogramme“ auf

Dr. Walter Lesch ist als Forscher des Schweizerischen Nationalfonds Oberassistent für Theologische Ethik an der Universität Fribourg.

1 Erweiterte Fassung eines Beitrags zum interuniversitären Forschungsprojekt „Film und Spiritualität" beim EUROCIC-Symposion in Luzern (10.-14. Juni 1992). Der essayistische Vortragsstil wurde beibehalten. Den Mitgliedern einer im Vorfeld dieser Veranstaltung konstituierten Arbeitsgruppe an der Universität Fribourg danke ich für anregende Diskussionen über Populärkultur und Fernsehserien.

2 Aus der Wahl dieses Mottos ist nicht auf weitergehende Übereinstimmungen mit den Ansichten des Autors der Father Brown-Geschichten zu schließen. 
dem Niveau des Insider-Kongreß-Tourismus bleiben und sich und die Theologie nicht durch weitergehende Ambitionen blamieren.

Dieser Skepsis ist freilich entgegenzuhalten, daß die Arbeit der kirchlichen Mediendienste den Vergleich mit anderen Medienfachstellen nicht scheuen muß. Sie findet Anerkennung, weil sie durch kompetente Filmkritiken und gut recherchierte Informationen überzeugt. Allerdings wird diese erfreuliche Einschätzung nicht selten durch den Einwand getrübt, eine solche Medienarbeit zeige zu wenig christliches bzw. theologisches Profil. Und dann beginnt normalerweise ein Streit, dessen Szenen schon in vielen Varianten, aber doch immer wieder mit ähnlichen Tendenzen und Polarisierungen durchgespielt wurden. Wo die einen die Autonomie der Kultur verteidigen, befürchten die anderen den Ausverkauf der Theologie. $^{3}$ Nun haben bekanntlich derartige Zuspitzungen den Nachteil, zu Halbwahrheiten zu werden, die sich leicht demontieren lassen. Wenn die Theologie sich z.B. auf Produkte der Populärkultur einläßt, verliert sich noch lange nicht ihr Profil, nur weil sie zur Analyse des Phänomens selbstverständlich auf sozial- und kulturwissenschaftliche Methoden zurückgreifen muß. Ich wüßte nicht, welche anderen Erkenntnisquellen ihr verfügbar wären. Wenn aber die refrainartige Berufung auf die Autonomie der ausdifferenzierten Kultursachbereiche zur Strategie der Immunisierung gegen lästige Kritik verkommt, dann kann mir das Lippenbekenntnis der Toleranz gestohlen bleiben. Es ist ein beliebter und offensichtlich gerade im Umgang mit Theologen erfolgreicher Trick, jede Kritik am ästhetischen Eigensinn von Kunstprodukten als banausenhaft abzuwehren. ${ }^{i}$ Zugegeben, die Theologie hat nach Jahrzehnten der Moralpredigt und der nach simplen Kriterien verfahrenden Bewertung von Kunst Grund genug, ein schlechtes Gewissen zu haben. Aber die Borniertheit von gestern und vorgestern kann heute nicht durch standpunktlose Offenheit kompensiert werden.

Konflikte werden oft dadurch umschifft, daß wir zum Zwecke der Dialoginszenierungen auf Filme zurückgreifen, die ohnehin mit theologischen Sympathien rechnen dürfen, weil sie thematisch irgendwie mit Religion in Verbindung zu bringen sind. Über die Filme eines Andrej Tarkowskij, z.B. NOSTALGHIA, läßt sich trefflich theologisieren; auch Wim Wenders' DER HIMMEL ÜBER BERLIN ist ein dankbares Objekt für theologische Assoziationen. ${ }^{5}$ Was aber machen wir, wenn uns im Kino oder vor dem Fernsehbildschirm ganz einfach nur die Wut überkommt angesichts des offensichtlichen Schwachsinns, den wir uns, zunächst noch geduldig und gesprächsbereit, angeschaut haben? Wie gehen wir mit solchen Irritationen um, wenn wir uns von der drohenden Etikettierung als Banausen nicht einschüchtern lassen wollen?

3 Jenen, die sich lange genug im Milieu katholisch-theologischer Ethik bewegt haben, ist das Repertoire solcher Gemeinplätze recht vertraut.

4 Martin Seel hat zur Verdeutlichung der besonderen Rationalität des Ästhetischen die Idealtypen des Ästheten und des Banausen als Extreme eines ästhetisch wenig idealen Verhaltens porträtiert. M. Seel, Die Kunst der Entzweiung. Zum Begriff der ästhetischen Rationalität, Frankfurt 1985, 315-317.

5 Ein gelungenes Beispiel für derartige Anstrengungen sind die schönen WendersInterpretationen in M. Kuhn/J. G. Hahn/H. Hoekstra (Hg.), Hinter den Augen ein eigenes Bild. Film und Spiritualität, Zürich 1991. 
Keine Angst! Nichts liegt mir ferner als eine kulturkonservative Schelte der angeblich grassierenden kulturellen Beliebigkeit. Die folgenden Überlegungen sind aus einer Irritation erwachsen: aus der Begegnung mit Filmen des amerikanischen Regisseurs David Lynch, der einem größeren Publikum vor allem durch die Fernsehserie TWIN PEAKS (1989-1991) bekannt geworden ist. Am Anfang meiner Seherfahrungen standen spontane Abneigung und der Trost, daß ich mir so etwas nicht um jeden Preis antun muß - auch nicht für ein interuniversitäres Forschungsprojekt. Die Abneigung wuchs beim Sehen einiger Kinofilme von Lynch und bei Gesprächen über diese Filme, bei denen sich meist verständnislose Kritiker und unerschütterliche Lynch-Fans gegenübersaßen. Genau diese Gesprächskonstellation hat bei mir Lernprozesse in Gang gesetzt und die Bereitschaft gefördert, doch noch einmal etwas genauer hinzuschauen und erste Urteile zu überdenken. Die hier skizzierten Gedanken beleuchten einige Aspekte dieses Prozesses, der mit der Abscheu vor Lynchs extremen Gewaltdarstellungen und vor einem, wie ich meine, fatalen Weltbild begann und in die Neugierde überging, zu begreifen, warum eine Serie wie TWIN PEAKS oder ein Film wie WILD AT HEART (1990) solche Begeisterung hervorrufen kann. Es handelt sich also weder um eine medienwissenschaftliche Analyse ${ }^{6}$ noch um eine theologische Interpretation, sondern um den Versuch einer Selbstvergewisserung angesichts von verunsicherten ethischen und ästhetischen Intuitionen.

\section{Dem Zeitgeist auf der Spur}

Unter den kompetenten FilmkennerInnen scheint Einigkeit darüber zu herrschen, daß TWIN PEAKS als Paradebeispiel für eine „postmoderne“ Fernsehserie gelten darf. Michel Bodmer kommt in einer Besprechung zu dem Ergebnis: „Jede Zeit hat die Fernsehserie, die sie verdient. ,Twin Peaks' ist gleichzeitig eine surreale und anspielungsreiche Seifenoper für die TV-Generation, ein Rotkäppchen-Märchen für das ,homo homini lupus'-Bewußtsein, ein postmoderner Psychokrimi für ein New Age, das die Vernunft nicht als alleiniges Mittel der Erkenntnis akzeptiert und bei alledem eine Serie, die immer wieder eine erfrischend ironische Subversion ihrer selbst betreibt. ${ }^{\text {"7 }}$ Endlich kommen wir dem Zeitgeist auf die Spur; endlich können wir uns am Bildschirm ein Bild davon machen, was bisher nur in nebulösen Texten kompliziert schreibender StarphilosophInnen zu lesen war. Die Postmoderne, ein Phantom des Feuilletons und akademischer Diskurse, ist ins Wohnzimmer eingekehrt und gibt sich die Ehre. ${ }^{8}$ Ein Gigant des Hollywood-Kinos, David Lynch, liefert den Peaks-Freaks den Stoff für gelehrte Gespräche über Mehrfachcodierungen in einer neuen Filmsprache, die sogar zur selbstironischen Relati-

6 Dazu gehörte sicherlich auch eine gründliche Erforschung des ökonomischen Umfelds der Produktion von Filmen und Fernsehserien und eine Rezeptionsanalyse. In ökonomischer Hinsicht sind die Marketing-Strategien des Lynch-Imperiums zur Flankierung der Twin Peaks-Serie besonders aufschlußreich. Eine CD mit der Filmmusik (Music from Twin Peaks), Bücher (The Secret Diary of Laura Palmer; The Autobiography of Special Agent Dale Cooper - beide wurden Taschenbuchbestseller), ein Reiseführer (Welcome to Twin Peaks), T-Shirts, Jacken und einige weitere Accessoires wurden parallel zur Serie erfolgreich auf den Markt gebracht.

7 M. Bodmer, Von Zwergen und anderen Riesen, in: ZOOM. Zeitschrift für Film 1/1992, 14f., hier 15. 
vierung fähig ist. ${ }^{9}$ Eine Serie, die sich über die Niederungen von DALLAS und DENVER erhebt - anspruchsvolle Unterhaltung!

Doch lassen wir diese Sticheleien beiseite, um uns der Frage zuzuwenden, wie scharf unser kulturdiagnostisches Analyseinstrumentarium eigentlich ist. Wie lassen sich einzelne Produkte der Gegenwartskultur mit größeren Trends in Verbindung bringen? Am Beispiel von TWIN PEAKS kann hier nur der bescheidene Versuch einer Antwort vorgelegt werden. Die Bezugnahme auf ein konkretes Produkt der aktuellen Massenkultur hat immerhin den Vorteil, abstrakte philosophische Diskurse auf den Boden heutiger Sehgewohnheiten holen zu können. Gerade das Werk von David Lynch ist ein gutes Beispiel für die Einebnung des Unterschieds zwischen elitärer Kunst und Populärkultur, Autorenfilm und Serienproduktion. ${ }^{10}$ Lynch hatte sich als exzentrischer Querdenker einen Namen gemacht - eine Art amerikanischer Peter Greenaway. Für die wenig innovative Gattung der TVSerie im Stil billiger Fließbandproduktion schien er nicht prädestiniert zu sein. Um so außergewöhnlicher war der Erfolg.

Unter den vielen Indizien für die gepriesene Postmodernität von Twin Peaks befindet sich ein Merkmal, das mit Lynchs grundsätzlichem Interesse an den dunklen Seiten des Menschen in Verbindung steht: die Faszination des Bösen, des Abgründigen und Geheimnisvollen, das in der Provinzstadt Twin Peaks verborgen ist und die FernsehzuschauerInnen fesselt. Vermutlich deshalb, weil es sich um Erlebnisdimensionen handelt, die in der Banalität unseres rationalen und rationalisierten Alltags gerade nicht vorkommen, von denen wir aber ahnen, daß sie unter der dünnen Oberfläche bürgerlicher Anständigkeit und Unauffälligkeit lauern können: große und kleine Verrücktheiten, kriminelle Neigungen, unterdrückte Begierden. Das gefundene Fressen für Moralisten, die eine genaue Vorstellung davon zu haben glauben, wie es eigentlich sein sollte.

David Lynch und sein Ko-Autor Mark Frost machen einen fiktiven Ort namens Twin Peaks (er soll in der nordöstlichen Ecke des Staates Washington in der Nähe der kanadischen Grenze liegen) zum Projektionsraum der alltäglichen und unheimlichen Ereignisse hinter der Fassade einer auf den ersten Blick ganz normalen amerikanischen Kleinstadt vor der pittoresken Kulisse der verschneiten Zwillingsgipfel, der Twin Peaks, die allerdings auch schon als Emblem der Doppeldeutigkeit verstanden werden können.

8 In den vergangenen Jahren habe ich mich bei verschiedenen Anlässen mit dem Postmoderne-Syndrom auseinandergesetzt, möchte jedoch die kulturdiagnostische Kraft dieser Debatte nicht überstrapazieren. Es geht nicht um die Propagierung eines neuen Zeitalters, sondern um die Sensibilierung für kulturelle Trends von größerer Tragweite. Vgl. W. Lesch, Medienethik unter „postmodernen“ Bedingungen, in: A. Holderegger (Hg.), Ethik der Medienkommunikation. Grundlagen, Fribourg/Freiburg i. Br. 1992, 73-103; W. Lesch/G. Schwind (Hg.), Das Ende der alten Gewißheiten. Theologische Auseinandersetzung mit der Postmoderne, Mainz 1993.

9 Es gibt innerhalb der Serie eine Serie, die die Bewohner von Twin Peaks gebannt am Fernsehschirm verfolgen ...

$10 \mathrm{Vgl}$. zur analogen Diskussion im literarischen Bereich den programmatischen Text von L. A. Fiedler, Überquert die Grenze, schließt den Graben! Über die Postmoderne, in: W. Welsch (Hg.), Wege aus der Moderne. Schlüsseltexte der Postmoderne-Diskussion, Weinheim 1988, 57-74. 
Der geniale Trick der TV-Serie besteht nun darin, daß trotz der vordergründigen Einkleidung eines Hauptstrangs der Handlung in eine Kriminalgeschichte das Böse seine damönische und mythische Kraft behält. FBI-Agent Dale Cooper kommt in die Provinz, um den Mord an Laura Palmer, einer Schülerin aus der Abschlußklasse der High School, aufzuklären und den vermuteten Zusammenhang mit einem Mordfall in einem anderen Bundesstaat zu untersuchen. Coopers ungewöhnliche Ermittlungsmethoden mit esoterischem Einschlag, sein Eintauchen in die Geheimnisse von Twin Peaks führen auf Umwegen zum Mörder: Leland Palmer, Lauras Vater, der vor seiner Verhaftung auch noch seine Nichte Maddy umbringt, die Laura zum Verwechseln ähnlich sieht (sie wird ebenfalls von der Laura-Darstellerin Sheryl Lee gespielt). Zu diesem Mörder gesellt sich in Träumen und Visionen jedoch noch eine weitere Figur, der Killer Bob, der angeblich Leland Palmer benutzt, um seine bösen Ziele zu erreichen. Die kriminalistisch zu untersuchende Horrorgeschichte von Inzest und Mord ist also auf einer zweiten Ebene die Geschichte der Besessenheit von einem bösen Damönen, der Leland ins Verbrechen und in den Wahnsinn treibt. In Folge 17 stirbt Leland in Coopers Armen in der Zelle der Sheriff-Station, nachdem er sich wie ein Bessener den Kopf an der Zellentür zertrümmert hat. Die bange Frage „Wo ist Bob jetzt?“ wird zum Leitmotiv für 13 weitere Folgen, in der sich Dale Cooper mehr und mehr in das Böse verstrickt.

Genau in dieser Hinsicht steht TWIN PEAKS in einer ästhetischen Tradition, die nicht voreilig in die Sprache der Ethik vermittelt werden sollte. Obwohl wir eigentlich wissen, daß es sich bei den dämonischen Abgründen um ein imaginiertes Böses handelt, geht von dessen Präsentation im Medium Film eine irritierende und provozierende Wirkung aus, die einer genaueren Untersuchung bedarf. Es geht nicht darum, an eine Fernsehserie eine moralisierende Meßlatte anzulegen. Das wäre banausenhaft, wie uns überzeugte Lynch-Fans mit Nachdruck erklären. Zu fragen wäre vielmehr nach der ästhetischen Konzeption des Geheimnisses von Twin Peaks, das nicht zuletzt auf einer ganz speziellen Polarität von Gut und Böse beruht, wobei das Böse in dieser Serie durchaus in einem metaphysischen Sinne aufgeladen ist, die über die moralische Qualifizierung von Handlungen oder Haltungen hinausgeht. ${ }^{11}$ Das Böse hat, wenn ich die Filme richtig interpretiere, die Qualität einer geheimnisvollen Macht, die von Menschen Besitz ergreift, sie besessen macht und in Abgründe der Gewalt und des Schreckens stürzt. Gemessen an den Charakteren der chaotischen und neurotischen Versager, mit denen Lynch Twin Peaks bevölkert und deren Umgangsformen auch schon ohne Dämonen ein gelungenes Abbild der Hölle wären, ist dies jedenfalls eine zusätzliche Dimension.

Ein theologisch-ethisches Erkenntnisinteresse bei der Filminterpretation bedarf der Klärung, um falsche Fragerichtungen zu vermeiden. Ethik verstehe ich nicht als Urteilsinstanz, die über Kunst richten will, sondern als hermeneutische Wissenschaft, die Handlungsorientierungen, insbesondere auch in den Medien, analysiert. Freilich bleibt die Frage nach dem eigenen Standpunkt und nach den

11 Alltagssprachlich verwenden wir die Vokabeln böse und falsch, gut und richtig meist nicht konsequent. Vgl. zur ethischen Terminologie: W. Lesch, Ethik und Moral / Gut und Böse / Richtig und Falsch, in: J.-P. Wils/D. Mieth (Hg.), Grundbegriffe der christlichen Ethik, Paderborn 1992 (UTB 1648), 64-83. 
eigenen ethischen und ästhetischen Präferenzen. Phänomene der Transgression im Medium der Kunst hat es zu allen Zeiten gegeben. Es war geradezu eine der Aufgaben von Kunst, Tabugrenzen zu verlagern und in imaginären Welten neue Handlungsspielräume zu eröffnen. Insofern drängt sich die ethische Befragung dieser Phänomene auch ohne ein verklemmtes Moralisieren fast von selbst auf.

Es sei schließlich noch daran erinnert, daß sich innerhalb der philosophischen Disziplin der Ästhetik in den vergangenen Jahren die Akzente in signifikanter Weise verlagert haben: vom Schönen zum Erhabenen. Die in klassischen Konzeptionen des Schönen (etwa bei Kant) noch enthaltene moralische Dimension tritt zurück zugunsten einer Steigerung von Gefühlsintensitäten..$^{12}$ Im Zusammenhang mit der Erfahrung von Chaos und Schrecken, von Gefahr und Geheimnis, von Gewalt und von überwältigenden Naturereignissen gewinnt die Thematisierung des Bösen eine neue Aktualität.

Als Zwischenbilanz läßt sich die von TWIN PEAKS ausgehende Irritation nun also etwas genauer angeben. Es ist nicht nur die Fülle der perfekt inszenierten Horroreffekte; es ist vor allem auch der mythologische Versuch einer "Erklärung" des Bösen in einem dualistischen Weltbild, das „aufgeklärte" Gemüter schockiert. Damit ist zunächst allerdings nur gesagt, daß Vorlieben und Abneigungen bei der Auswahl vom Filmen etwas über die eigenen Idiosynkrasien aussagen. Wer sich der Gewalt in ästhetischer Distanzierung aussetzt, ist noch nicht selbst gewalttätig. Andererseits gibt es den verständlichen Reflex, auf Gewaltdarstellungen allergisch zu reagieren. Wer beim Einschalten der Fernsehnachrichten mit Bildern des Grauens überflutet wird, ist zumindest skeptisch, wenn ihn dieses Medium zum Zweck der Unterhaltung auch noch mit fiktiven Szenen der Gewalt konfrontiert. Auf die breite wissenschaftliche Diskussion über den Konsum solcher Medienprodukte möchte ich hier nicht eingehen..$^{13}$ Offensichtlich ist aber gerade die Ohnmacht angesichts des realen Bösen auf dem Balkan oder des neuen Rassismus in Deutschland ein Argument gegen den optimistischen Glauben an Aufklärung und moralische Entwicklung. Trotz vielfacher Anstrengungen und Interventionen bleiben die Abgründe der Grausamkeit ein erklärungsbedürftiges Phänomen und wecken das Interesse an den fiktiven Darstellungen des Extremen, die gewiß eine psychische Entlastungsfunktion haben können. Haben sie aber auch einen Erkenntniswert? Oder ist diese Frage schon falsch gestellt?

\section{Die Imagination des Bösen und die „permanente Theodizee“}

Die theoretische Ausgrenzung des Bösen hat in der philosophischen Tradition eine lange Geschichte und bereitet auch in der gegenwärtigen Ästhetik und Ethik (ganz zu schweigen von der Theologie) einige Mühe. ${ }^{14}$ Nicht zufällig führen diese Bemühungen immer wieder in den Bereich der Kunst: „Die Verwissenschaft-

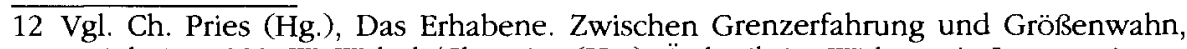
Weinheim 1989; W. Welsch/Ch. Pries (Hg.), Ästhetik im Widerstreit. Interventionen zum Werk von Jean-François Lyotard, Weinheim 1991.

13 Einen guten Überblick vermittelt das Kapitel "Gewalt in Trivialromanen und im Film“ in U. Rauchfleisch, Allgegenwart von Gewalt, Göttingen 1992, 104-146, bes. $141 \mathrm{ff}$. 
lichung des Bösen provoziert ein Komplement: Die Ästhetisierung des Bösen. Die Ästhetisierung ist eine Folge der Verwissenschaftlichung: Indem das und der Böse darstellbar wird, wird er auf eine gewisse Weise verfügbar - und wo das Böse begrifflich nicht faßbar wird, springt die Kunst ein. Was - wie immer - darstellbar ist, verliert den Schrecken des Unnennbaren, es wird das dargestellte Böse in seiner Bosheit greifbar, es gerät in eine Art elementare, mythische Verfügbarkeit. ${ }^{15}$

In einem stark beachteten Aufsatz hat Karl Heinz Bohrer 1985 drei idealtypische Reaktionen auf das Böse in der Kunst folgendermaßen porträtiert: „Wenn der Pädagoge A nach Anhörung eines Vortrags über Kleists Selbstmord als imaginatives Projekt meint, diese Katastrophe müsse doch vom Vortragenden vermittelt werden; wenn der Philosoph B anläßlich einer Diskussion über Friedrich Schlegels Absage an die Geschichtsphilosophie zugunsten einer ästhetischen Utopie sich erregt, wer derlei gedanklich nachvollziehe, sei ein Anwalt des Bösen; und wenn der Soziologe $\mathrm{C}$ schließlich eine Versöhnung herstellt zwischen Aufklärung und Romantik, dann haben alle drei, der mahnende Pädagoge, der zürnende Philosoph und der vermittelnde Soziologe, einen Beleg dafür gebracht, inwiefern der künstlerisch-literarischen Rede für den akademisch-politischen Menschen immer noch ein Skandal innewohnt, der, wie aufgeräumt sich Pädagogen, Philosophen und Soziologen im allgemeinen auch gegenüber dieser Sphäre des Scheins zu verhalten wissen, bis heute nicht gezähmt werden konnte bzw. mit unrichtigen theoretischen Vorschlägen zu zähmen versucht wird. “16

Das Unheimliche des Bösen soll also gezähmt werden durch pädagogische Vermittlung, die das Katastrophale, eventuell auch das Krankhafte eines abweichenden Verhaltens (Suizid) unterstreicht; durch die Aufhebung der Eigendynamik des Schrecklichen in einem geschichtsphilosophischen (heilsgeschichtlichen?) Gesamtkonzept; durch die Integration des auf den ersten Blick Irrationalen in das aufklärerische Projekt der vernünftigen Weltanschauung. Nur der Schlaf der Vernunft gebiert Ungeheuer; also darf die Vernunft nicht einschlummern, sondern muß in grenzenloser Wachheit die Schatten des Bösen bekämpfen. Es ist wohl nicht zuletzt dieser titanische Kampf gegen das „Andere“ der Vernunft und für die „permanente Theodizee" 17 - auch im ästhetischen Medium -, die zur Erlahmung der kreativen Energien der Moderne beigetragen hat, in deren Folgen so etwas wie eine postmoderne Grundstimmung überhaupt erst entstehen konnte. Eine so verstandene Postmoderne wäre nicht unbedingt eine neue Epoche, sondern eine zu allen Zeiten mögliche Relativierung der ideologiekritischen Aufklärung.

Gerade über die Relativierung des "Projekts der Moderne" wurde aber im vergangenen Jahrzehnt in deutschen Intellektuellenkreisen erbittert gestritten. Die

14 Vgl. die Dokumentation einer Berliner Ringvorlesung vom Wintersemester 1989/90 in C. Colpe/W. Schmidt-Biggemann (Hg.), Das Böse. Eine historische Phänomenologie des Unerklärlichen, Frankfurt 1993.

15 Schmidt-Biggemann, ebd., 11.

16 K. H. Bohrer, Das Böse - eine ästhetische Kategorie?, in: Merkur 39 (1985) Heft 6, 459473 , hier 459.

17 Vgl. K. H. Bohrer, Die permanente Theodizee. Über das verfehlte Böse im deutschen Bewußtsein, in: Merkur 41 (1987) Heft 4, 267-286. 
Gründe für die Ausgrenzung des auch nur imaginierten Bösen in Deutschland nach 1945 sind evident. Nach einer Zeit des faschistischen Mythosmißbrauchs ist es eine naheliegende Reaktion, jeden ästhetischen Rückgriff auf den Mythos zunächst einmal unter den Verdacht antiaufklärerischer Regression zu stellen, um den gefürchteten Einbruch des Bösen in die Rationalität zu bannen. Aufgeklärte ästhetische Rationalität wäre folglich immmer sozial und ethisch gebändigt und ihrer dunklen Seiten beraubt.

In dem grob skizzierten Sinn einer an moralischen Standards orientierten Modernitätstheorie ist TWIN PEAKS in der Tat postmodern: eine soziale Botschaft soll nicht vermittelt werden. Das Geheimnis von Twin Peaks löst sich nicht sozialoder psychoanalytisch auf. Gibt dies nun Anlaß zur Klage über die „Zerstörung der Vernunft" in einer schwarzen Kunst? Ist es angebracht, vor den Folgen der Imagination des ästhetisch Bösen zu warnen? Oder wären dies nun vergebliche Versuche, das Irrationale zu verdrängen, dabei aber auch das Kunstwerk zu zerstören?

Einige geschichtliche Hinweise zu dieser „schwarzen“ Tradition in der modernen Kunst mögen den Konflikt der Interpretationen und Wertungen verdeutlichen. Ein auch von Bohrer gerne zitiertes Paradebeispiel ist Flauberts historischer Roman SALAMMBÔ (1862), in dem der Autor in konsequenter Durchführung seines Grundsatzes der „impassibilité“ (Unempfindlichkeit) sadistische Szenen der Grausamkeit (bis hin zu Kinderopfern an den Sonnengott Moloch) in Karthago nach dem 1. Punischen Krieg entwirft. Mit derartigen Wahrnehmungsschocks, der aus der Verunsicherung konventioneller Ästhetik und Moral entstehen, experimentierten die literarischen Avantgarden der Folgezeit mit ihren Zerstörungsphantasien. ${ }^{18}$ Die Schwierigkeiten bei der Deutung historischer Gestalten einer irritierenden Kunst wiederholen sich in der heutigen Verlegenheit angesichts der Grausamkeit mancher Bilder in Kino, Fernsehen und Video. ${ }^{19}$ Verteufelungen dieser Visualisierungen des Schrecklichen und Grausamen sind wohl ebenso unangebracht wie unkritische Tendenzen, voreilig einen "Zuwachs an ästhetischer Autonomie“ zu loben. Wir werden zunächst zugeben müssen, daß Psychothriller zu den attraktivsten Kinogenres gehören, weil sie Verdrängtes ans Licht bringen und die Bodenlosigkeit der Existenz drastisch schildern. ${ }^{20}$

Versuche, das Irrationale zu bannen, indem wir es als irrational abstempeln ${ }^{21}$, führen auf längere Sicht doch nur zu einer Rückkehr des Verdrängten. In neueren Diskussionen zur Hermeneutik wurde darauf aufmerksam gemacht, daß unsere Suche nach Wahrheit und Weisheit auf schwankendem Boden steht. Wie Jochen Hörisch in einem Essay herausgestellt hat, entpuppt sich Hermes als der doppelgesichtige Patron der Hermeneutik und der Hermetik: „Die olympischen

18 Vgl. die psychoanalytische Interpretation von J. Kristeva, Pouvoirs de l'horreur. Essai sur l'abjection, Paris 1983. Ferner: G. Bataille, La littérature et le mal, Paris 1957.

19 Vgl. H. Hartwig, Die Grausamkeit der Bilder. Horror und Faszination in alten und neuen Medien, Weinheim/Berlin 1986.

$20 \mathrm{Vgl}$. die Hinweise auf klassische und neuere Kinofilme in F. Derendinger, Psychothriller: Befreiung der Schattenseite, in: ZOOM. Zeitschrift für Film 4/1993, 12-20.

$21 \mathrm{Vgl}$. U. Eco, Das Irrationale gestern und heute, in: ders., Im Labyrinth der Vernunft. Texte über Kunst und Zeichen, Leipzig 21989, 376-390. 
Götter gelten nicht eben als sonderlich seriös. Betrügen, berauben und überlisten sie doch die Sterblichen wie ihre unsterblichen Mitgötter in einem Übermaß, das verläßliches Hören, Sehen und Verstehen vergehen macht. Unseriöser ist aber unter ihnen keiner als ausgerechnet der Gott, dessen Aufgabe es ist, gesicherte Informationsübermittlung und verständigen Umgang miteinander zu gewährleisten: Hermes. Keine Schandtat, die ihm nicht zuzutrauen wäre. Was nicht ausschließt, ihn als Genie des Betrugs und der systematischen Desinformation auch zu bewundern. ${ }^{\text {“22 }}$ Hermes baut mit dem Panzer einer Schildkröte eine Leier - ein Opfer für die Kunst! „Eine hermeneutische und hermetische Urszene. Eine Urszene, die gleißend verdeutlicht, daß das Schreckliche der Anfang der ästhetischen Regel ist, danach das Schöne nichts als des Schrecklichen Anfang ist. "23

Diesen Aspekt der Mehrdeutigkeit hat auch Umberto Eco unterstrichen: „Fasziniert vom Unendlichen entwickelt die griechische Kultur, neben dem Begriff der Identität und Widerspruchsfreiheit, die Idee der fortwährenden Metamorphose, symbolisiert durch Hermes. Hermes ist ungreifbar, volatil, doppelgesichtig, Patron aller Künste, aber auch der Diebe, iuvenis et senex, Jüngling und Greis zugleich. Im Mythos von Hermes werden die Prinzipien der Identität, der Widerspruchsfreiheit und des ausgeschlossenen Dritten negiert, die Kausalketten winden sich um und über sich selbst zu Spiralen, das Nachher geht dem Vorher voraus, der Gott kennt keine räumlichen Grenzen und kann in verschiedenen Formen an verschiedenen Orten gleichzeitig sein." ${ }^{\text {"2 }}$

Insofern überrascht es nicht, wenn wir heute ein neues Interesse an der spätantiken (Multi-)Kultur beobachten können. Im der rückblickenden Konstruktion erweist sich jene Zeit der Mysterienkulte und des Synkretismus als eine Art antike „Postmoderne“. In diesen Zusammenhang gehört auch das gnostische Erbe, das Böse als Offenbarungserfahrung zu zelebrieren. Wenn alles Geheimnis ist und der Mensch Opfer eines kosmischen Komplotts werden kann, dann gewinnt die Einweihung in kosmische Mysterien spielerische oder gar existentielle Attraktivität. Dale Cooper aus TWIN PEAKS ist mit seinem Hang zur Esoterik ein Guru derartiger Initiation.

Jedes theologische Sprechen von Mysterium und Offenbarung müßte sich der Rezeptionsbedingungen vergewissern, die heute durch massenmediale Einflüsse geprägt werden. Das theologische Raunen vom „Geheimnis“ hat zwar nicht erst in unseren Tagen Konkurrenz bekommen; es ist aber immer neuen Mißverständnissen ausgesetzt, die bei einer fortschreitenden kulturellen Akzeptanz esoterischer Praktiken leicht entstehen können.

\section{Die Suche nach dem Mörder}

Einer der wichtigsten Praktiker und Theoretiker postmoderner Ästhetik ist Umberto Eco, der in der Nachschrift zu seinem Erfolgsroman DER NAME DER ROSE sein eigenes literarisches Programm mit den Anliegen postmoderner Litera-

22 J. Hörisch, Die Wut des Verstehens. Zur Kritik der Hermeneutik, Frankfurt 1988, 9.

23 Ebd., 10.8

24 Eco (Anm. 21), 379.0 
turtheorie verknüpft hat. Mit Leslie A. Fiedler vertritt er die These, „daß die Träume der Leser zu bevölkern nicht unbedingt heißen muß, sie zu besänftigen, mit versöhnlichen Bildern zu trösten. Es kann auch heißen, sie aufzuschrecken: mit Alpträumen, Obsessionen. “25 Nichts anderes ist das erklärte Ziel von Lynch und seinem Ko-Autor Frost.

Freilich bliebe zu fragen, inwieweit sich gerade die Gattung des Kriminalromans, des aufklärerischen Modells par excellence, für die Realisierung einer aufklärungskritischen Sinnverweigerung eignet. Bei Eco ist schließlich der tüchtige Detektiv erfolgreich und bringt den diabolischen Bösewicht zur Strecke ${ }^{26}$ In TWIN PEAKS geht die Rechnung nicht so glatt auf; der Kampf zwischen Gut und Böse endet im Alptraum eines labyrinthischen Verwechslungsspiels.

Ein Beispiel für die Vorliebe des Aufklärers für eindeutige Verhältnisse im Krimi lieferte Jürgen Habermas ganz nebenbei in einem Vortrag auf einem Philosophenkongreß. Für seinen Geschmack läßt der niederländische Erfolgsautor Janwillem van de Wetering in seinem Roman RATTENFANG „den roten Faden seiner Fabel im Wirrwarr abwechslungsreicher Kontexte so weit zerfasern, bis der gattungsspezifische Unterschied zwischen Täter und Opfer im Gewebe der vielen kleinen Differenzen unkenntlich wird - und die Polizei nach verständnisvoller Aussprache mit dem endlich ertappten, aber sympathischen Mörder auf Anzeige und Strafverfolgung verzichtet. “27 Diese Kurzinterpretation wäre natürlich im Detail zu überprüfen. Aber sie ist symptomatisch für die Weigerung, sich auf ästhetische Experimente mit unseren Schattenseiten wirklich einzulassen. Das beliebte Krimi-Genre in Literatur und Film hat noch längst nicht all seine Möglichkeiten ausgereizt. Für den ethischen Diskurs ist es - nicht nur aus didaktischen Gründen ein interessantes Untersuchungsobjekt, und zwar gerade dann, wenn die klare Polarität von Gut und Böse verloren geht: in den Figuren der Verbrecher und der Ermittler, in den Strukturen der Medienöffentlichkeit und der Justiz. ${ }^{28}$

\section{Die Traumwelten des David Lynch}

Der folgende Blick auf Bilder des Bösen bei David Lynch bezieht sich vor allem auf die TV-Serie TWIN PEAKS (1989-1991), ferner auf die Kinofilme WILD AT HEART (1990) und TWIN PEAKS - FIRE WALK WITH ME (1992). Im Twin PeaksFilm wird die Vorgeschichte zum Plot der Serie erzählt. ${ }^{29}$ Ich beschränke mich auf diesen kleinen Ausschnitt aus Lynchs Werk, möchte aber betonen, daß es seit den ersten großen Arbeiten dieses Regisseurs eine erstaunliche Konstanz in der Vorliebe für schockierende Bilder aufweist. ERASERHEAD (1977), THE ELEPHANT MAN

25 U. Eco, Nachschrift zum „Namen der Rose“, München 41984, 82.

26 Vgl, dazu: U. Schulz-Buschhaus, Kriminalroman und Post-Avantgarde, in: Merkur 41 (1987) Heft 4, 287-296. Zum Überblick über die Gattung: U. Leonhardt, Mord ist ihr Beruf. Eine Geschichte des Kriminalromans, München 1990.

27 J. Habermas, Die Einheit der Vernunft in der Vielheit ihrer Stimmen, in: ders., Nachmetaphysisches Denken. Philosophische Aufsätze, Frankfurt 1988, 153-186, hier 180.

28 Von großer ethischer Relevanz sind verschiedene Aufsätze in dem spannenden Buch von L. Böllinger/R. Lautmann ( Hg.), Vom Guten, das noch stets das Böse schafft. Kriminalwissenschaftliche Essays zu Ehren von Herbert Jäger, Frankfurt 1993. 
(1980), DUNE (1984) und BLUE VELVET (1986) sind zu Kultfilmen einer eingeschworenen Fan-Gemeinde geworden.

Unübersehbar sind die traditionellen Krimi-Elemente in TWIN PEAKS: mit dem üblichen Personal der Ermittlung in einem dunklen Fall, in dem Mord, Prostitution, Inzest, Drogenhandel und Immobilienspekulation eine Rolle spielen. Allein das in Medien und Forschung stark beachtete Inzest-Thema ${ }^{30}$ wäre ein Anlaß für ausführliche Interpretationen. Wir könnten sogar die These aufstellen, daß Lynch mit der Entlarvung der Perversionen in einer heilen Provinzwelt ein genuin aufklärerisches Anliegen vertritt. Die Angst vor der Aggression des Vaters als zentrales Motiv in TWIN PEAKS steht in scharfem Kontrast zur Idylle von Douglastannen, Kaffee und Kirschkuchen. Die Institutionen der Kleinstadt (Kirche, Schule) haben ihre Glaubwürdigkeit längst verspielt; Jugendliche und Erwachsene bewegen sich orientierungslos durch die privaten und öffentlichen Räume ihrer Stadt und ihrer geheimnisvollen Umgebung.

Der Film wird untermalt von der effektvollen Musik des Komponjsten Angelo Badalamenti, der den einzelnen Figuren der Serie ihre Themen und Stimmungszustände zuordnet. Wichtig war für Lynch die Wahl des Drehortes in der Berglandschaft im Nordwesten der USA (NORTHWEST PASSAGE lautete der Arbeitstitel der Serie), deren Atmosphäre im Vorspann eingefangen wird: ein Vogel auf einem Zweig, ein Sägewerk und Nahaufnahmen seiner Maschinen, das Ortseingangsschild "Welcome to Twin Peaks., Population 51.201" und ein Wasserfall in der Nähe des Ortes - das sind die Bilder, die zusammen mit Badalamentis langsamer Titelmelodie zu Beginn jener Serienfolge auf die Geheimnisse einer 24Stunden-Handlung einstimmen.

Eine Besonderheit der Serien sind die bizarren Traumsequenzen, in denen in einem merkwürdigen Raum mit roten Vorhängen Hinweise auf Laura Palmers Mörder gegeben werden. Die suggestiven Bilder mit ihren bizarren Gestalten und Farben habe eine beinahe hypnotische. Wirkung und durchbrechen die Konventionen des realistischen Erzählkinos.

Wertvolle Interpretationshilfen sind die von Robert Fischer gesammelten Selbstaussagen von David Lynch, der freimütig über seine Absichten und Obsessionen Auskunft erteilt: ${ }^{31}$ „Ich glaube, daß Filme Kraft haben sollten. Die Kraft des Guten und die Kraft des Bösen. So,daß man berührt wird und die Verhältnisse in Bewegung geraten. Wenn man Angst hat, das zu riskieren, endet alles in einer lauwarmen Soße." ${ }^{\text {"32 }}$ "Ich finde es höchst interessant, wie der Humor in unmittelba-

29 Vgl. R. Fischer, Twin Peaks - Fire Walk with me, in: ZOOM. Zeitschrift für Film 8/ 1992, 28f.

30 Vgl. Rauchfleisch (Anm. 13), 79-93.

31 Von „Abstakt" bis „Zucker": Ein Lynch-Porträt in Selbstaussagen, in: R. Fischer, David Lynch. Die dunkle Seite der Seele, München 1992 (Heyne Filmbibliothek 32/165), $246 \mathrm{ff}$. Ich verdanke diesem informativen und glänzend geschriebenen Buch wichtige Anregungen.

32 Ebd., 253. 
rer Nachbarschaft mit der Gewalt und dem Horror des Lebens existiert. Die Nähe dieser nur scheinbaren Gegensätze reizt mich. Und ich habe begonnen, damit spielerischer umzugehen. ${ }^{333}$

Lynch äußert sich auch zu seinen künstlerischen Vorbildern in Film, Malerei und Literatur (Hitchcock, der Surrealismus, Francis Bacon, Kafka) und bestätigt damit einige Vermutungen, die sich beim ersten Sehen aufdrängen. Er stellt sich ausdrücklich in eine Tradition der ästhetischen Moderne, die wir im Zusammenhang mit Bohrers Schockästhetik des Bösen kennengelernt haben. Die dort geäuBerten Thesen haben also auch für das künstlerische Schaffen von Lynch einen (wenn auch begrenzten) Erklärungswert.

Es bleibt die Frage nach der mythologischen Grundstruktur von Lynchs Imagination des Bösen. Läßt sich das dualistische Konstruktionsprinzip präzise nachweisen? Im Kinofilm TWIN PEAKS - FIRE WALK WITH ME hat Lynch wieder mit der dämonischen Figur des Bob gearbeitet; allerdings erscheint auch das Gute als personifizierte Traumgestalt: als Lauras Schutzengel auf einem kitschigen Bild an der Wand ihres Zimmers, der zunächst aus dem Bild verschwindet und dann in der Schlußsequenz zur Musik von Luigi Cherubini von der Decke schwebt. Dämonen und Schutzengel sind also ironische Hilfskonstruktionen aus dem Repertoire volkstümlicher Vorstellungen; sie verharmlosen aber nicht den realen Terror, den Leland Palmer durch den sexuellen Mißbrauch seiner Tochter ausübt.

1990 wurde Lynch in Cannes für seinen Film WILD AT HEART mit der Goldenen Palme ausgezeichnet und erntete dafür in der Filmkritik ähnlich enthusiastische und empörte Reaktionen wie bei TWIN PEAKS. Das Road Movie erzählt die Geschichte von Sailor und Lula, die sich leidenschaftlich lieben, jedoch von Lulas Mutter Marietta auseinandergebrachtwerden sollen. ${ }^{34}$ Nach Szenen brutalster Gewalt und ohrenbetäubendem Sound endet der Film mit einem Happy End unter dem Schutz der guten Fee aus dem populären Kinderbuch THE WIZARD OF OZ und mit Elvis Presleys LOVE ME TENDER, Lulas Lieblingssong. Also wieder eine postmoderne Mixtur? Im Feuilleton der „Zeit“ schwärmte Andreas Kilb: „Lynchs Kino ist der vollkommene amerikanische Alptraum: perfekt, pervers, postmodern. ${ }^{\prime 35}$ Andere Kritiker verglichen WILD AT HEART mit Steven Spielbergs INDIANA JONES AND THE TEMPLE OF DOOM, jenem Spektakel für die Mittelklasse, während Lynch auf ein aufgeklärt-dekadentes Publikum setze. ${ }^{36}$

\section{Macht des Mythos - Ohnmacht theologischer Ethik?}

Lynchs Filme führen den faszinierten oder irritierten Zuschauer in die Welt der alten und neuen Mythen Amerikas, aus denen imaginäre Welten gebastelt werden. Der Erfolg dieser Fiktionen wird nicht zuletzt damit zusammenhängen, daß sie den Nerv gegenwärtiger kultureller Befindlichkeit ziemlich genau treffen.

33 Ebd., 258.

$34 \mathrm{Vgl}$. Fischers Lynch-Monographie, 190-216.

35 Zit. n. Fischer, ebd., 211.

36 Diese Stimmen aus der amerikanischen Filmkritik werden ebenfalls referiert in Fischer, ebd., 215. 
Nobert Bolz hat diese mythenfreundliche Stimmung folgendermaßen charakterisiert: „Mythen sind zergliederte, wohlstrukturierte Gesamtheiten, die doch als aus Resten zusammengesetzt sich erweisen. Unter der Hand des Bastlers Mythos wandelt sich Flickwerk in Struktur. Sie organisiert Elemente unterschiedlicher Herkunft zu Geschichten, deren Buntheit sich verlockend von der grauen Theorie abendländischer Vernunft abhebt. Heute tritt dem Betrachter das gefährliche Denken in mythischer Verpuppung entgegen. Begnügt er sich nicht mit der ästhetischen Lust an der mythischen Textur, so vermag er versuchen, die Herkunft des Gedankenstoffs zu erforschen, von dem der Mythos stets nur Enden, Reste, Flicken ,aufhebt ${ }^{6}$ und kühn neuorganisiert: des Stoffs, aus dem die Reden des Poststrukturalismus montiert sind. So scheint der eschatologischen Zeit der Linken - der Zeit der kurzen Wege, des ,Umschlags" von Theorie und Praxis, des Endes der Interpretation - fast zwangsläufig eine neue Mythologie zu folgen: Umwege des Erzählens, Entlastung von der Geschichte durch Geschichten; statt des linken ,Geschichtemachens ${ }^{, 37}$

Eine entmythologisierte Theologie kann auf einen solchen Trend nicht gleichgültig reagieren. Es wäre zu einfach, die „postmodernen“ Filmmythen nur als Kontrastfolie zu benutzen: als Bilder einer erlösungsbedürftigen Welt, der die christliche Erzählung von Gelingen und Versöhnung als Therapie anzubieten wäre. Trotzdem bleibt die Herausforderung durch die neuen Bilder, auch wenn alles nur Fiktion sein sollte. Bei aller ironischen Brechung sind Lynchs Filme aber gerade nicht nur referenzlose Zeichenkompositionen; zu den Phantasien der Zerstörung, Zerstückelung und Vergewaltigung und den Bildern des Bluts und des Feuers gibt es grausame Entsprechungen in der Realität. Und insofern fangen sie durchaus etwas von dem Kontext ein, in dem wir fast ohnmächtig versuchen, Geschichten gegen die Gewalt zu erzählen. Wie können wir heute noch die christliche Heilsgeschichte erzählen? Als Erfolgsstory? Als Geschichte vom Gerechten, der getötet wurde? Dessen gewaltsamer Tod übrigens zugleich als kulturgeschichtliche Ermöglichung einer Ästhetik des Häßlichen gedeutet werden kann?

All dies hat Konsequenzen für die ethische Analyse von postmoderner Filmästhetik. Ein möglicher Weg wäre die ideologiekritische Auseinandersetzung mit David Lynch als einem Repräsentanten der reaktionären Reagan-Bush-Ära, die angeblich von der Sehnsucht nach den 50er Jahren und vom Dualismus von Gut und Böse geprägt war. Die Sache scheint aber viel komplizierter zu sein und zwingt uns, als verärgerte oder ratlose Zuschauer einen zweiten Blick zu wagen. Wenn es Lynch nicht gelingt, die Gewalt ästhetisch zu bannen, so berechtigt dies noch nicht zum moralischen Verdikt über Lynchs Filmkunst. Für unsere Schwierigkeiten mit der alltäglichen Gewalt in ihren brutalen und subtilen Ausprägungen werden wir keinen Rat im Kino oder vor dem Fernsehbildschirm erwarten dürfen. Aber wir werden Lynch nicht anlasten können, daß auch er nicht weiß, warum Menschen hassen, morden, quälen, erniedrigen. Lynchs ästhetische Konzeption des Bösen steht in enger Verbindung zur literarischen und filmischen Tradition dieser Schattenseite der Vernunft und führt die Schockästhetik konsequent weiter.

37 N. W. Bolz, odds and ends. Vom Menschen zum Mythos, in: K. H. Bohrer (Hg.), Mythos und Moderne. Begriff und Bild einer Rekonstruktion, Frankfurt 1983, 471492 , hier 471 
Ob dies eine Schocktherapie sein kann, vermag ich im Moment nicht zu entscheiden. Es gab beim Sehen der Filme Momente, in denen ich am liebsten jede weitere Beschäftigung mit diesen Kunstprodukten abgebrochen hätte. Die vorsichtig sondierende Sicht in die Abgründe der eigenen Rezeptionshaltung müßte jetzt eigentlich erst beginnen. In Abgrenzung zur ästhetizistischen Isolierung der Kategorie des Bösen erlaube ich mir die Frage, ob die Medienbilder nicht doch ihre Unschuld verlieren können und als trivialisierte Ikonen der Gewalt zur Erosion zivilgesellschaftlicher Verständigung beitragen. ${ }^{38}$ "Postmoderne" Theoretiker mögen dies als naive Harmoniesucht abtun und ihre Spottlieder über den heuchlerischen Diskurs der Menschenrechte anstimmen ${ }^{39}$; eine überzeugende Antwort auf die Gegenwart der Gewalt haben sie aber bisher nicht gegeben.

Nicht ohne Unbehagen blicke ich zurück auf das Patchwork der vorgetragenen Gedanken, die nach Systematisierung und Fortsetzung verlangen. Dennoch schließe ich hier in der Hoffnung, wenigstens einige Diskussionsimpulse für ein noch zu führendes Gespräch zwischen Theologie und Filmkritik über die Verschränkung von ethischen und ästhetischen Wertungen gegeben zu haben. ${ }^{40}$

SUMMARY: The aesthetics of the evil as an principle of "postmodern" style? Theologic-ethical remarks on the TV series of Twin Peaks

The theological interest in film has its limits, when pictures of brutality and violence and skillful productions of the evil thward any benevolent receptiveness. In the name of the autonomy of aestetic imagination we got used to condemn such pictures not in a rash by a moral verdict; but the radical separation of aesthetic and moral judgements seems to be impossible without contradiction. With the TVseries of Twin peaks as an example and some glances on movies by David Lynch, the author tries on a theologicethical level to reflect on the confusions created by „postmodern“ film myths.

RÉSUMÉ: L'esthétique du mal en tant que principe de style „postmoderne"? Note marginale éthico-théologique à propos de la série télévisée „Twin peaks“

L'intérêt théologique apporté au film rencontre une limite quand des images de brutalité, de violence et de vraie mise en scène du mal viennent contrarier une attitude réceptive bienveillanté. Au nom de l'autonomie d'une imagination esthétique, nous nous sommes certes habitués à ne pas soumettre à la légère de telles images à un verdict moral, toutefois, une séparation radicale des valeurs esthétiques et morales ne semblent pas non plus être possible sans contradiction. En se référant à la série télévisée „Twin Peaks“ et en jetant un coup d'oeil sur les films cinématographiques de David Lynch, on va essayer de refléter au niveau éthico-théologique les irritations qui proviennent des mythes cinématographiques „postmodernes".

38 Vgl. zum Zweifel an der Unschuld radikaler Imagination: Hartwig (Anm. 19), 165f.

39 So etwa J. Baudrillard, Transparenz des Bösen. Ein Essay über extreme Phänomene, Berlin 1992, 100ff.

$40 \mathrm{Vgl}$. den anregenden Bd. 4 der vom Gemeinschaftswerk der Evangelischen Publizistik e. V. herausgebenen Arnoldshainer Filmgespräche: Die Ästhetik des Bösen im Film, Frankfurt 1987. 
RESUMEN: La estética de la maldad como principio ,postmoderno'? Observaciones teológico-éticas a la serie de TV Twin Peaks

El interés teológico en la película llega a su fin cuando imágenes de brutalidad y violencia y hábiles escenificaciones de la maldad entrecruzan imágenes beneficiosas. Aunque nos hemos acostumbrado, en nombre de la autonomía de la imaginación estéti$\mathrm{ca}$, a no someter tales imágenes a un veredicto moral apresurado, sin embargo parece no ser posible sin un contradicción una separación radical de los valores estéticos y morales. Tomando como ejemplo la serie de TV Twin Peaks y echando una mirada a las películas de David Lynch se intenta reflexionar sobre las irritaciones teológico-éticas surgidas a partir de los mítos fílmicos ,postmodernos'. 